\title{
In-patient care for children with type 1 diabetes across hospitals in the Yorkshire and Humber region in the north of England
}

N Amin, S Uday, F Campbell, J Yong

Department of Paediatric Diabetes, Leeds Children's Hospital, Leeds, UK.

\section{Introduction}

An important part of diabetes management is maintaining high standards of in-patient care. The first inpatient standards for management of children with diabetes were set and audited in the South of England in 2011'.

Deficiencies highlighted were: lack of dietetic advice on wards, lack of education sessions for ED and ward staff and lack of contact with diabetes team especially for overnight admissions

\section{Aims}

Identification of variations in in-patient care provided to children with type 1 diabetes across the Yorkshire and Humber region, with a view to standardisation of care.

\section{Methods}

The audit was conducted against in-patient care standards identified by the Children and Young Person's Diabetes Implementation Support Group (CYPDISG). Questionnaires were sent to clinical leads of all paediatric diabetes units in the region, which serves a total of 2599 children and young people with diabetes.

\section{Results}

Sixty three per cent of the units, consisting of 2 tertiary and 8 secondary care units, responded. Paediatric wards and EDs in all units had protocols for management of new diagnosis of diabetes, diabetic ketoacidosis (DKA), hypoglycaemia and surgery. The following table illustrates availability of protocols.

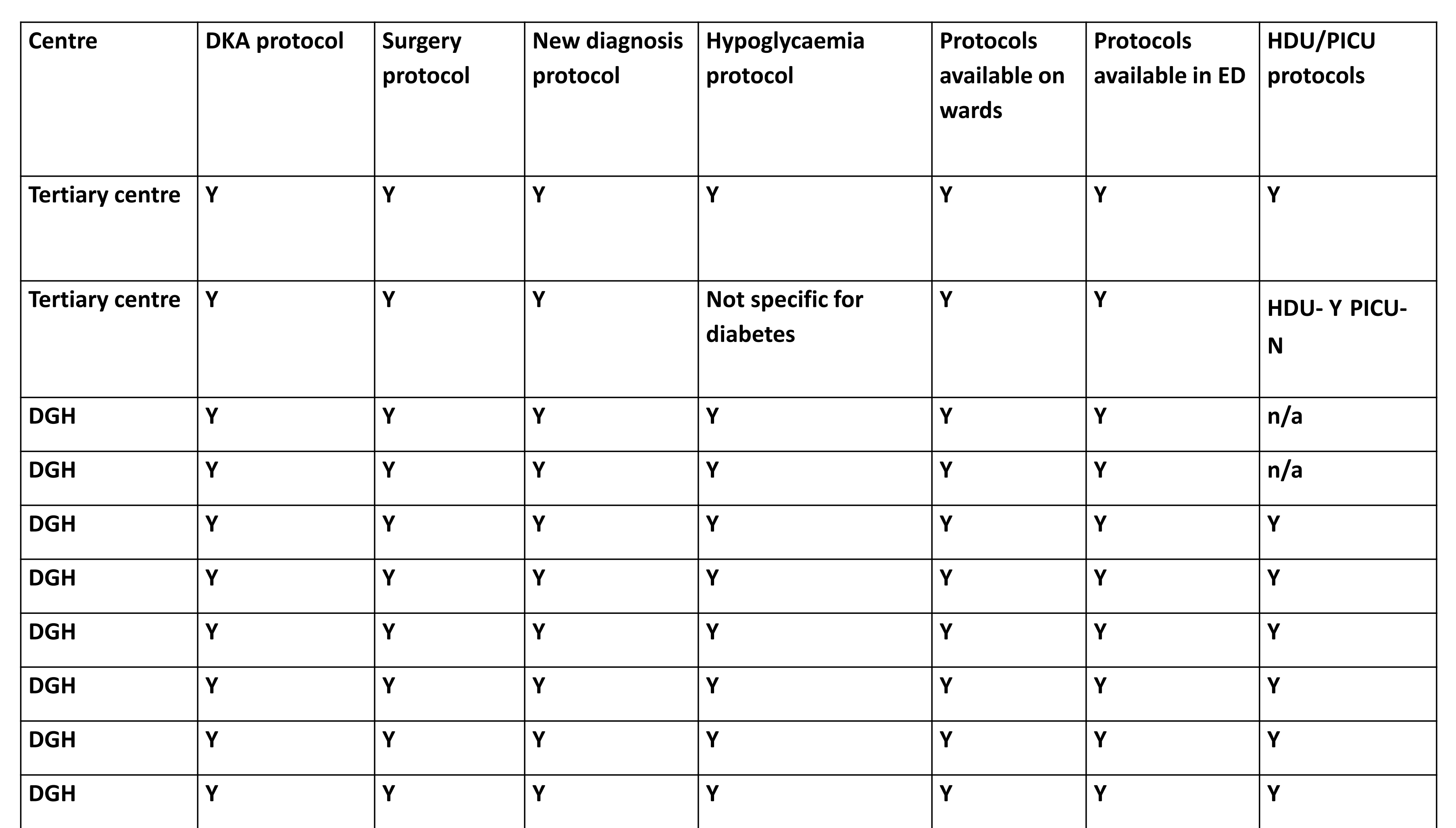

\section{Results}

Nine out of 10 units had paediatric nurses in areas where children were cared for, but only the tertiary centres had a trained paediatric nurse in the emergency department (ED) on every shift. A 24 hour on-call service was only provided by $40 \%$ of the units. The diabetes team was usually contacted within 2 hours of an admission in tertiary centres and within 24 hours in secondary care units. Paediatric diabetes specialist nurses (DSN) had an active role in in-patient management in all units.

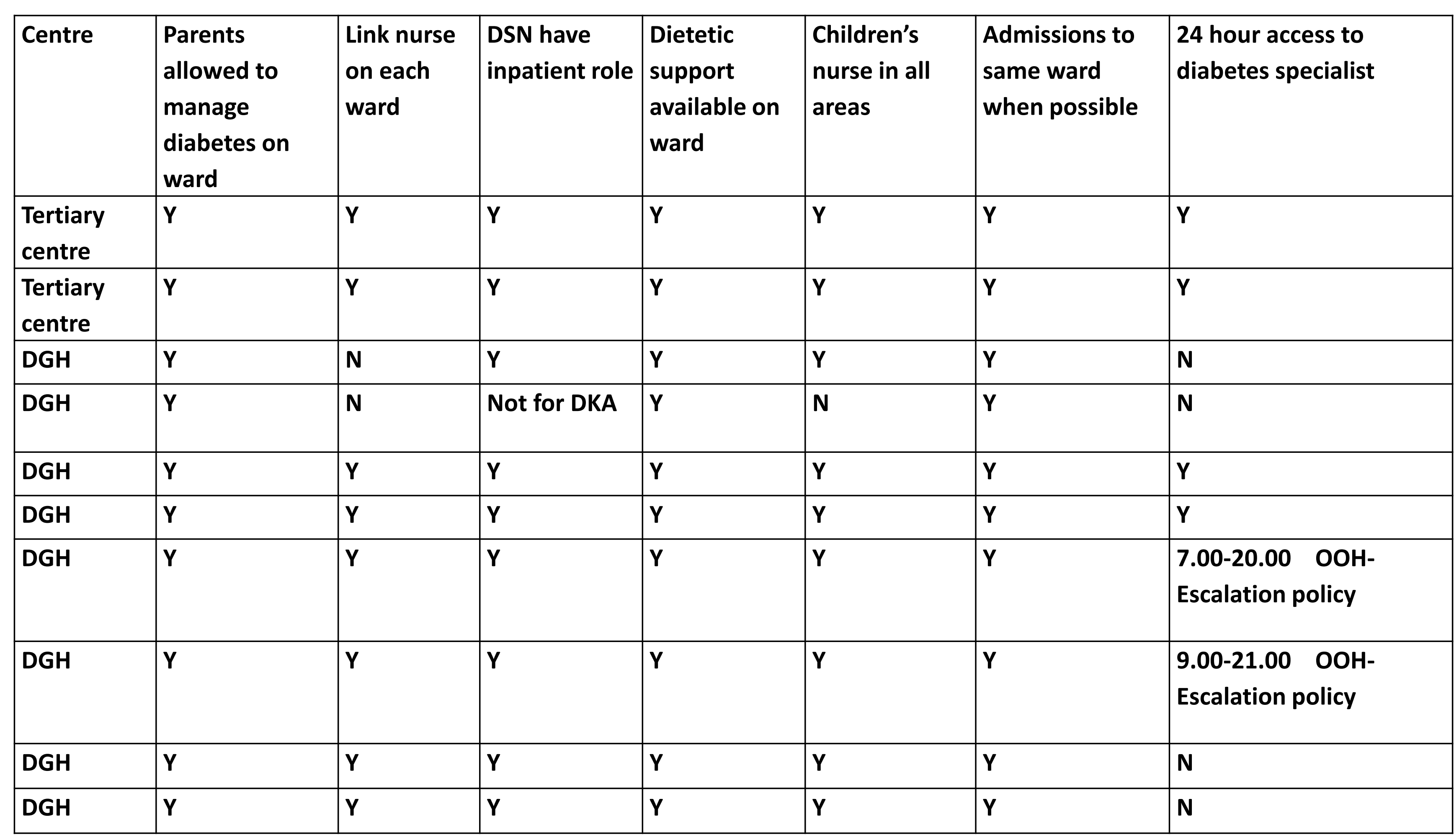

All units had regular education sessions for ward staff, although $20 \%$ expressed concerns regarding poor attendance and $50 \%$ of units had education sessions for ED staff. Only 2 units had insulin prescription charts and only tertiary centres routinely audited insulin prescription and administration errors.

\begin{tabular}{|l|l|l|l|l|l|}
\hline Centre & $\begin{array}{l}\text { Insulin prescription } \\
\text { charts }\end{array}$ & $\begin{array}{l}\text { Audit of insulin } \\
\text { prescription errors }\end{array}$ & $\begin{array}{l}\text { Education- ED } \\
\text { staff }\end{array}$ & Liaison with ED & $\begin{array}{l}\text { Regular education } \\
\text { sessions for ward } \\
\text { staff }\end{array}$ \\
\hline Tertiary centre & N & Y & Y & Y & Y \\
\hline Tertiary centre & Y & Y & Y & Y & Y \\
\hline DGH & N & N & Y & Y & Y \\
\hline DGH & N & N & Y & N & Y \\
\hline DGH & N & N & N & Y & Y \\
\hline DGH & N & N & N & Y & Y \\
\hline DGH & N & N & N & Y & Y \\
\hline DGH & Y & N & Y & Y & Y \\
\hline DGH & N & N & N & Y & Y \\
\hline DGH & N & N & N & Y & Y \\
\hline
\end{tabular}

\section{Conclusions}

This audit demonstrates on-going difficulties achieving current standards of in-patient care for children and young people with diabetes. There is a lack of 24 hour on-call service in majority of the paediatric diabetes units. There needs to be standardisation across the region and feasibility of implementation needs to be explored. 